\title{
Human Antigen Presenting Cell Gene Array Profiling of the Effect of Human T Cell Leukemia Virus Type I Tax Protein on Dendritic Cells
}

\author{
Suman Datta*‡, Pooja Jain, Jaya Ahuja and Brian Wigdahl
}

Address: Department of Microbiology and Immunology, and Institute for Molecular Medicine and Infectious Disease, Drexel University College of Medicine, Philadelphia, PA, USA

* Corresponding author $\ddagger$ Presenting author

from 2005 International Meeting of The Institute of Human Virology

Baltimore, USA, 29 August - 2 September 2005

Published: 8 December 2005

Retrovirology 2005, 2(Suppl I):P25 doi:10.1 I86/I742-4690-2-SI-P25

HTLV-1-associated myelopathy/tropical spastic paraparesis (HAM/TSP) is characterized by a highly stimulated immune response including the oligoclonal expansion of CD8+ cytotoxic T lymphocytes specific for viral oncoprotein Tax. Studies have demonstrated that Tax may be available for immune recognition by dendritic cells (DCs). In this study, a pathway-specific human dendritic and antigen presenting cell gene array has been used to study the global transcriptional changes mediated by extracellular Tax on monocyte-derived dendritic cells (MDDCs). Of the 192 genes examined, approximately 100 genes were differentially expressed after extracellular exposure to Tax. These genes were functionally categorized as the genes involved in antigen uptake and presentation (MHC class II molecule: HLA-DMB, DC-SIGN), intracellular adhesion molecules (ICAM-1 and -2), T cell costimulatory molecule (ICOS), cell surface receptor (CD47, toll-like receptors: TLR-1, -3, -6, -9), and several cytokines, chemokines; and their receptors (IFN-g, IL-6, IL-12, TNF- $\alpha$, IL-17, CCL5, CCL20, TNFSF4). Additionally, the expression of cellular signal transduction genes: ATF4, NFKB1, RELA, RELB (members of the NFkB family), G1P2 and G1P3 (interferon inducible) was also significantly altered. The expression of DC activation markers was confirmed by real-time PCR and was in direct correlation with the microarray observations. 